\title{
Inclined convection in a layer of liquid water with poorly conducting boundaries
}

\author{
Stefano Castellini $\odot,{ }^{1}$ Marina Carpineti $\odot,{ }^{1}$ Fabrizio Croccolo $\odot,{ }^{2}$ and Alberto Vailati ${ }^{1, *}$ \\ ${ }^{1}$ Dipartimento di Fisica A. Pontremoli, Università degli Studi di Milano, I-20133 Milano, Italy \\ ${ }^{2}$ Universite de Pau et des Pays de l'Adour, E2S UPPA, CNRS, TOTAL, LFCR UMR5150, Anglet, France
}

(Received 19 December 2019; accepted 10 September 2020; published 24 September 2020)

\begin{abstract}
We investigate pattern formation in an inclined layer of liquid water with poorly conducting boundaries. We show that above the threshold for convection the presence of an inclination larger than 14 mrad determines a transition from a square pattern to longitudinal rolls, a behavior remarkably different than the one reported in the presence of inclined conducting boundaries, where transitions between convective planforms occur at inclination angles of the order of several degrees. The longitudinal rolls are characterized by a dimensionless wave vector $k \approx 1.8$, significantly smaller than $k \approx 3.117$ reported for conducting boundaries at large Prandtl number. The transition can be triggered by changing dynamically the inclination of the layer of fluid, and does not occur symmetrically in the two directions. By starting in the horizontal configuration, it develops slowly through the demolition of the square structure to form longitudinal rolls, while it develops rapidly in the other direction, through the formation of a cross-roll structure perpendicular to the longitudinal rolls.
\end{abstract}

DOI: 10.1103/PhysRevResearch.2.033481

\section{INTRODUCTION}

A horizontal layer of fluid under the action of a temperature difference $\Delta T$ represents an archetypal model for the understanding of pattern formation in nonequilibrium systems [1]. When the fluid is heated from below and the temperature difference between the boundaries exceeds a threshold value, a macroscopic convective flow develops inside the fluid and, through a symmetry breaking mechanism, gives rise to selforganized convective patterns [2]. Significantly, the system can be parametrized entirely in terms of the dimensionless Rayleigh number $\mathrm{Ra}=\alpha g \Delta T h^{3} /(v \chi)$ and Prandtl number $\operatorname{Pr}=v / \chi$, where $\alpha$ is the thermal expansion coefficient, $g$ is the acceleration of gravity, $v$ the kinematic viscosity, $h$ is the thickness of the sample, and $\chi$ the thermal diffusivity. By adopting dimensionless units the same set of equations can be used to describe pattern formation across a wide range of length scales, from a few microns up to astrophysical distances. The geometry and symmetry of the patterns formed are strongly influenced by the boundary conditions. Most of the research on convection in single component fluids has been performed in the presence of ideal boundary conditions, such as horizontal layers of fluid and perfectly conducting boundaries [3]. However, the cases where conditions are not ideal have a remarkable relevance for the understanding of the behavior of natural systems, where the boundaries are in general not horizontal and conduct heat poorly. For example, masses of water [thermal conductivity

\footnotetext{
*alberto.vailati@unimi.it

Published by the American Physical Society under the terms of the Creative Commons Attribution 4.0 International license. Further distribution of this work must maintain attribution to the author(s) and the published article's title, journal citation, and DOI.
}

$\left.\kappa_{w}=0.60 \mathrm{~W} /(\mathrm{m} \mathrm{K})\right]$ at the Earth's surface are frequently bound by silicates at the bottom $\left[\kappa_{s}=3 \mathrm{~W} /(\mathrm{m} \mathrm{K})\right]$ and air at the top $\left[\kappa_{a}=2.6 \times 10^{-2} \mathrm{~W} /(\mathrm{m} \mathrm{K})\right]$, which conduct heat poorly. Therefore, a deeper understanding of the role of poorly conducting - nonhorizontal-boundaries is of great interest for the modeling of convective heat transfer in natural systems as oceans and the atmosphere.

A first important model system to understand the influence of nonideal boundaries is represented by an inclined layer of fluid with conducting boundaries. In the absence of inclination the pattern formation process is entirely determined by the buoyancy force, which close to the threshold of the instability $\mathrm{Ra}_{c}=1708$, gives rise to randomly oriented parallel rolls with wave number $k=3.117$. An inclination of the layer determines the onset of a large-scale flow (LSF), where the upper half of the layer flows downhill parallel to the upper boundary in the direction of inclination, while the bottom half of the layer flows uphill, parallel to the lower boundary. The LSF determines a shear stress on the layer of fluid, which strongly affects the convective patterns generated by buoyancy in the absence of inclination. A first effect of the tilt of the liquid layer is that for small inclination angles the convective rolls align parallel to the direction of inclination [4-7]. At larger inclination angles, a transition from longitudinal rolls to transverse rolls occurs [6,7]. Depending on the inclination angle, the Rayleigh number, and the Prandtl number, a complex scenario of convective patterns appears $[8,9]$. This scenario comprises dynamical configurations such as Busse oscillations, subharmonic oscillations, undulation chaos, longitudinal and transverse bursts [6,10-17]. Under these conditions, transitions between patterns are observed at large inclination angles, at least of the order of a few degrees, when the component of the gravity force parallel to the layer of liquid becomes large enough to promote a strong LSF, able to compete effectively with the vertical flow determined by Rayleigh-Bénard convection. 
A notable exception to this behavior occurs in binary liquid mixtures, where even an inclination of the sample as small as few milliradians strongly affects the convective planform [18-22]. This feature of binary mixtures can be qualitatively understood by taking into account that in the case of solutal convection the boundaries are impermeable. Therefore, a perturbation of the concentration field cannot be dissipated by the boundaries and persists for a long time, thus determining a large scale flow even for small inclinations.

A second relevant case of nonideal boundary conditions for Rayleigh-Bénard convection in a single component fluid is represented by poorly conducting boundaries. In this case experiments [23,24] and theoretical modeling [25-28] have shown that when the Prandtl number is large enough $(\operatorname{Pr} \geqslant 1)$ and the thermal conductivity of the boundaries is comparable or smaller than that of the fluid, the convective planform is made by square cells, which represent the configuration that maximizes the transfer of heat [25]. The wave number $k$ of the instability is strongly influenced by the thickness and thermal conductivity of the boundaries in relation to those of the sample and varies in an ample range, the two limiting cases being represented by perfectly conducting boundaries $(k=3.117)$ and perfectly insulating boundaries $(k=0)$. A convective square planform has been reported also for solutal convection in a binary liquid mixture, where the impermeable boundaries are formally analog to perfectly insulating boundaries in the thermal case. So far, the influence of inclination on pattern formation with impermeable or poorly conducting boundaries has been investigated mostly in binary liquid mixtures [18-20,22], but not in single component fluids with poorly conducting boundaries.

In this work we investigate the pattern formation process in a layer of liquid water with poorly conducting boundaries. We show that when the layer is horizontal the convective planform is made of square cells of wave number $k=1.8 \pm 0.15$, in agreement with theoretical predictions of Riahi [28]. We show that close to the threshold for convection and in the presence of inclination angles larger than the critical angle $\delta_{c}=14$ $\mathrm{mrad}$ the pattern is made of longitudinal rolls aligned parallel to the direction of inclination. The transition between the two structures can be obtained by varying continuously the inclination of the liquid layer, and displays hysteresis. By starting with a horizontal layer of liquid, the imposition of an inclination larger than $\delta_{c}=14 \mathrm{mrad}$ determines the demolition of the square pattern to create the longitudinal rolls. Conversely, by removing the inclination in the presence of longitudinal rolls, a cross roll structure develops perpendicularly to them, and gives rise to the square pattern. The two transition processes occur with well separated timescales, the first being a factor 3 slower than the second one.

\section{EXPERIMENT}

The sample is a layer of ultra-pure water (Chem-Lab H2OLF $1 \mu \mathrm{S}, 0.4 \mu \mathrm{m}$ UV filtered) confined laterally by an O-ring with an inner diameter $\Phi=49 \mathrm{~mm}$ and sandwiched between two plexiglass windows with a diameter of $75 \mathrm{~mm}$ and a thickness of $5.15 \mathrm{~mm}$ (Fig. 1). The O-ring determines a lateral boundary that is only approximately cylindrical. Indeed, the lateral surface bounding the sample has a slight hourglass

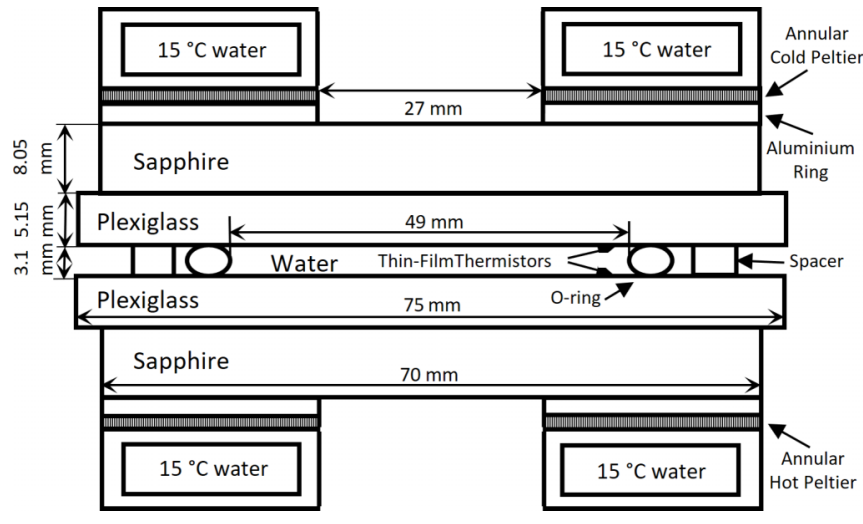

FIG. 1. Cross section of the thermal gradient cell. The two sapphire windows guarantee a uniform horizontal temperature distribution at the outer surface of the plexiglass windows, which confine a layer of liquid water in the vertical direction. The temperature difference imposed to the liquid layer is measured by using two thin-film thermistors thermally coupled to the inner surface of the plexiglass windows.

shape. However, due to the strong compression of the O-ring, the hourglass shape is not pronounced. We estimate that the maximum variation of the diameter of the sample as a functions of height is less than 5\%. This allows us to assume that the small deviation of the lateral boundary from a cylindrical shape does not influence the patterns significantly. The use of a circular lateral boundary guarantees that the square and roll patterns are not forced by a preferential orientation determined by the geometric shape of the lateral boundary, as it would happen in the presence of a rectangular geometry. The distance between the two plexiglass windows is determined by three calibrated spacers made of Delrin, with a thickness $h=3.10 \mathrm{~mm}$. The relatively high aspect ratio $\Gamma=\Phi /(2 h)=$ 7.9 of the sample guarantees that the lateral boundaries do not affect significantly pattern formation at the center of the sample. The thermal conductivity of the plexiglass plates used to bound the sample is $\kappa_{p}=0.18 \mathrm{~W} /(\mathrm{m} \mathrm{K})$, while for water $\kappa_{w}=0.60 \mathrm{~W} /(\mathrm{m} \mathrm{K})$. Following previous theoretical work [25-28], the two dimensionless parameters that characterize the boundaries are the ratio of the thermal conductivities of the boundaries and of the sample $\zeta=0.30$, and the ratio $\lambda=3.3$ between the overall thickness of the boundaries and that of the sample. The fact that the sample and the boundaries have thermal conductivities of the same order of magnitude determines a deep penetration of the temperature field inside the plexiglass windows. The typical distance beyond which the imprint of the temperature field determined by the convective patterns is not felt any more inside the boundaries is of the order of the sample thickness $h$. The choice of boundaries with a thickness larger than $h$ and a thermal conductivity smaller than that of the sample guarantees that the boundaries behave as poor conductors of heat [23].

The temperature of the sides of the plexiglass windows in contact with the sample is measured by two thin-film $10 \mathrm{k} \Omega$ thermistors, thermally coupled to the windows. Each of the two plexiglass windows is thermally and optically coupled to a sapphire window of thickness $8.05 \mathrm{~mm}$ and diameter of $70 \mathrm{~mm}$ by means of a thin layer of silicone oil with a 
viscosity of $300 \mathrm{cSt}$ (Dow Corning FS 1265). The temperature of the sapphire windows is controlled by two annular Thermo Electric Devices (TED), with an inner aperture that guarantees optical access to a circular region with a diameter of $27 \mathrm{~mm}$. One side of each TED is thermally coupled to a sapphire window by means of an aluminium ring, while the other is in contact with a annular flange kept at a constant temperature of $15^{\circ}$ by means of a water circulation thermostat. Due to their high thermal conductivity $\kappa_{s}=36 \mathrm{~W} /(\mathrm{m} \mathrm{K})$ the sapphire windows guarantee a uniform temperature distribution on the sides of the plexiglass windows not in contact with the sample. Convective patterns are visualized by using the shadowgraph projection method [29]. All the optical components and the cell are fixed to a vertical optical bench rail with a length of $1.5 \mathrm{~m}$, which can be inclined with an accuracy of 0.1 mrad by using tilt screws and a comparator to read the tilt angle. The leveling of the system is calibrated and checked regularly by using a precision frame level with an accuracy of $20 \mu \mathrm{rad}$. In this way, the optical diagnostics and the thermal gradient cell can be tilted as a whole, and this avoids optical deformations of the visualized convective patterns. The light source is a super-luminous light emitting diode (Superlum SLD 261-MP-DIL-SM-PD) with a wavelength of $676.5 \mathrm{~nm}$ and a bandwidth of $14.1 \mathrm{~nm}$, coupled to a monomode optical fiber. The output of the fiber lies in the focal plane of an achromatic doublet of focal length $500 \mathrm{~mm}$, which collimates the light onto the cell. An achromatic doublet with a focal length of $300 \mathrm{~mm}$ placed after the cell conjugates a plane located at a distance $z=135 \mathrm{~cm}$ from the sample onto a Jai-CVM300 charge coupled array detector (CCD) with a resolution of $768 \times 576$ pixels. The defocussing distance $z$ allows to work in a shadow configuration, where the propagation of light transforms phase modulations determined by the convective structures into amplitude modulations that can be recorded by the CCD [29,30]. A reference image taken in the absence of a temperature gradient was subtracted to each shadowgraph image to get rid of the optical background determined by spatial inhomogeneities in the illumination of the sample.

The features of the convective pattern depend on the Rayleigh number, the Prandtl number, and the boundary conditions. In our case the Prandtl number is fixed at $\operatorname{Pr}=7$, while the Rayleigh number and the boundary conditions can be varied. More in detail, as far as the boundary conditions are concerned, the aspect ratio $\Gamma=7.9$ and the ratio of the thermal conductivity of the plexiglass windows and that of the water sample are fixed, while the inclination $\delta$ of the liquid layer can be changed as described above.

\section{RESULTS}

After leveling accurately the cell in the horizontal position, we performed a set of test measurements aimed at determining the experimental conditions needed to generate square patterns. An external temperature difference in the range 20-60 K was applied to the sample, and the effective temperature difference imposed to the sample was recorded by using the two thin-film thermistors. After a transient time of the order of $5000 \mathrm{~s}$ the rearrangement of the convective patterns gave rise to stable square patterns for Rayleigh numbers in the range $1500<\mathrm{Ra}<2800$ [Fig. 2(a)], the upper limit being

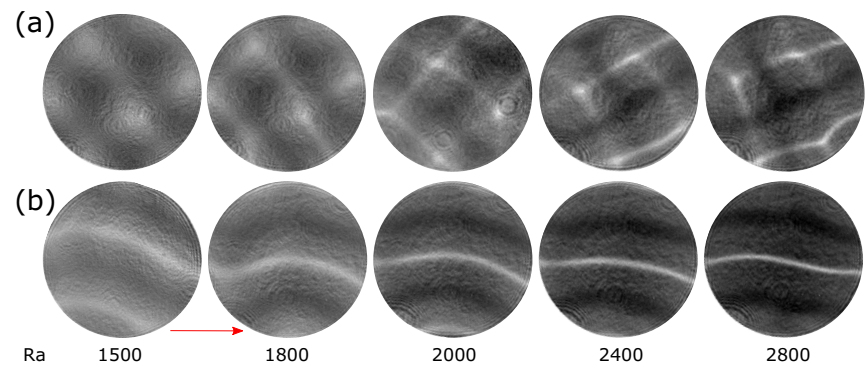

FIG. 2. Square patterns and longitudinal rolls formed in the absence and in the presence of inclination. (a) Stationary square patterns; (b) stationary longitudinal rolls formed in the presence of an inclination of $27 \mathrm{mrad}$. The arrow marks the projection of the direction of steepest ascent of the boundaries. The diameter of each panel corresponds to $27 \mathrm{~mm}$.

determined by the performances of the TEDs. The stability of the square patterns in the absence of inclination was checked by performing measurements lasting as long as $12 \mathrm{~h}$.

A similar set of measurements was performed by imposing an inclination angle of $27 \mathrm{mrad}$ and applying to the sample the same temperature differences used to obtain the square pattern. Under these conditions, the patterns exhibited a longitudinal roll structure, aligned parallel to the direction of inclination [Fig. 2(b)].

\section{A. Phase space}

We investigated systematically the geometry of the convective patterns by preparing the sample at a fixed inclination and suddenly applying a stable temperature difference to it. The time needed for the stabilization of the vertical temperature profile through the boundaries and the sample is of the order of $\tau_{T} \approx 2800 \mathrm{~s}$.

After a stabilization time of the order of $5000 \mathrm{~s}$, of the same order of magnitude of $\tau_{T}$, the patterns reached a stable configuration characterized either by the formation of a square pattern, or by longitudinal rolls aligned parallel to the direction of inclination of the sample. Figure 3 summarizes the phase space of the convective patterns, as a function of the Rayleigh number and inclination angle. Patterns become visible when the Rayleigh number exceeds the critical value $\mathrm{Ra}_{c i}=1300$, smaller that the critical Rayleigh number $\mathrm{Ra}_{c}=$ 1708 associated to Rayleigh-Bénard convection with conductive boundaries. From Fig. 3 one can appreciate the presence of a transition between square and roll patterns, occurring at a critical value of the inclination angle of the order of $\delta_{c} \approx 14$ mrad for Rayleigh numbers $\mathrm{Ra}<2400$. This transition occurs by increasing the inclination angle $\delta=13 \mathrm{mrad}$ by about 2 mrad. This results in a tiny increase, of the order of $g / 500$, of the in-plane component of the acceleration of gravity $g \sin (\delta) \approx g / 80$. Measurements performed in the absence of inclination allowed us to perform a direct comparison with the dimensionless wave number of square structures determined theoretically for a horizontal layer of fluid with $\lambda=1[26,27]$ and $\lambda=\infty[25,28]$ [Fig. 4(a)]. From the comparison one can appreciate that our experimental result $k=1.8 \pm 0.15$, obtained with $\lambda=3.3$, is fully compatible with the theoretical result $k=1.76$, obtained for $\lambda=\infty[25,28]$. Conversely, our 


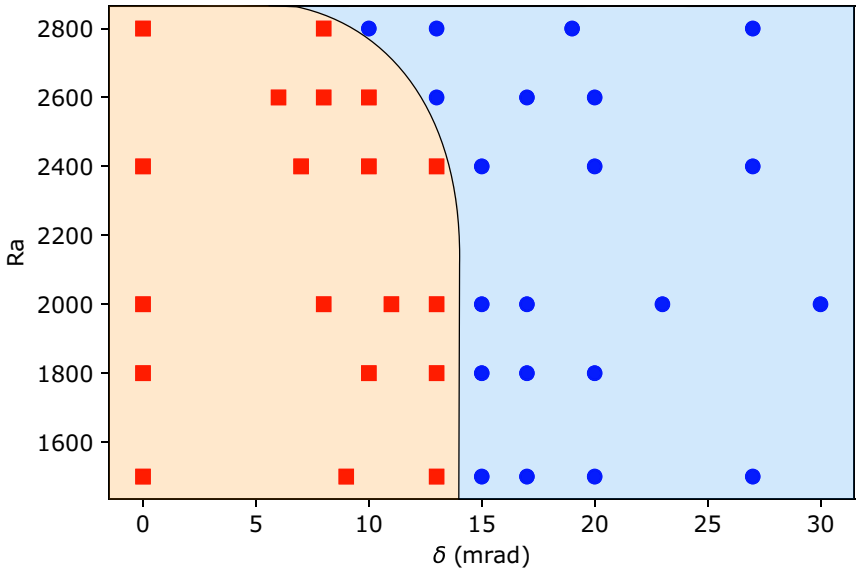

FIG. 3. Phase space. Geometry of the patterns plotted as a function of the inclination angle and of the Rayleigh number. Square symbols indicate square patterns, while circles represent longitudinal rolls. One can appreciate the presence of the transition between the two geometries, qualitatively indicated by the solid line separating the amber and a light blue regions.

results is not compatible with the theoretical value $k=2.16$ obtained for $\lambda=1$ [26,27]. These results suggest that a thickness ratio $\lambda=3.3$ like the one adopted by us is large enough to mimic effectively the ideal condition of a insulating boundaries of infinite thickness.

Previous experiments reported square structures obtained in single components liquids with poorly conductive boundaries. However, these experiments were performed with boundary conditions that do not allow a fully quantitative comparison with theoretical predictions. The first evidence of (a)

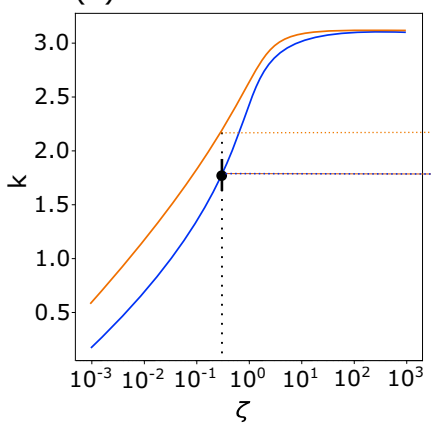

(b)

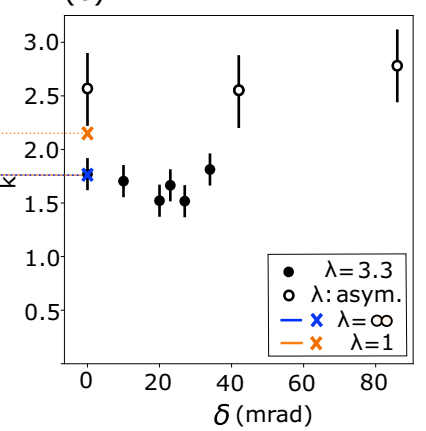

FIG. 4. Wave number of convective patterns. (a) Wave number of the pattern in the absence of inclination as a function of the ratio $\zeta$ of the thermal conductivities of the boundaries and of the sample. The orange and blue lines are the theoretical predictions of Proctor $(\lambda=1)$ [26,27], and Riahi $(\lambda=\infty)$ [28], respectively. Full circles in panels (a) and (b) correspond to the wave numbers of square patterns measured by us in water $(\zeta=0.3, \lambda=3.3$ and $\operatorname{Pr}=7.0)$ averaged in the range of Rayleigh numbers $1500 \leqslant \mathrm{Ra} \leqslant 2800$. (b) Wave number of the convective patterns plotted as a function of the inclination angle. The orange and blue crosses represent the extrapolation at $\zeta=0.3$ of the theoretical curves shown in panel (a). Open circles are the experimental results by Shadid and Goldstein [24] for ethylene glycol with $\operatorname{Pr}=90$ and asymmetric boundaries made by the superposition of layers of poorly conducting materials. a square pattern was obtained by Legal and Croquette for a sample of liquid water $(\operatorname{Pr}=7)$ [23]. They found $k=2.5$ and they attempted a qualitative comparison with the theoretical predictions by Proctor [26] and Riahi [28]. A quantitative comparison was prevented by the complex boundary conditions adopted in the experiment, which made a straightforward estimation of $\zeta$ and $\lambda$ not possible: A 4.0-mm-thick sample of water was bound by two plexiglass windows with a thickness of $8.0 \mathrm{~mm}$ sandwiched by two glass windows of thickness $12.7 \mathrm{~mm}$ flushed by thermostated water that allowed to control independently the temperature of each window. Shadid and Goldstein reported square structures in a sample of ethylene glycol $(\operatorname{Pr}=90)$ [24]. They used a thermosensitive liquid crystal sheet to visualize the convective structures. This method allowed them to perform systematic measurements of the wave number of the square structures as a function of inclination angle. In the absence of inclination the wave number was found to be $k=2.6 \pm 0.35$, fully compatible with the experimental result by Legal and Croquette [23]. However, the clever visualization method chosen by Shadid and Goldstein required to adopt asymmetric boundary conditions, where the bottom part of the fluid was in contact with a complex sandwich made by a transparent mylar sheet $(0.10 \mathrm{~mm})$, a liquid-crystal layer $(0.10 \mathrm{~mm})$, a polyester film coated with gold $(0.12 \mathrm{~mm})$, a thick lexan layer $(22 \mathrm{~mm})$, and a thick extruded polystyrene layer $(50.8 \mathrm{~mm})$; the top of the liquid layer was in contact with a high strength glass plate flushed with thermostated water. The complex geometry adopted in the experiment by Shadid and Goldstein also prevents the determination of the thermal conductivity ratio $\zeta$ and of thickness ratio $\lambda$, thus making a fully quantitative comparison with theoretical predictions impossible. To our knowledge, our results represent the first quantitative experimental confirmation of the theoretical model developed by Riahi for Rayleigh-Bénard convection in single component fluids with poorly conducting boundaries [28].

\section{B. Wave number}

We investigated systematically the dependence of the wave number from the inclination angle. Measurements performed by changing the inclination of the sample from 0 to $40 \mathrm{mrad}$ showed that the wave number varies in the range $1.5<k<$ 1.8 , and is not significantly affected by the transition from square patterns to longitudinal rolls occurring at $\delta \approx 14 \mathrm{mrad}$ [Fig. 4(b)]. As far as we know, the only other experimental results for convection in an inclined single component liquid with poorly conducting have been obtained by Shadid and Goldstein in the experiments with ethylene glycol $(\mathrm{Pr}=90)$ detailed above [24]. In the range of very small inclination angles explored by us they found $2.5<k<2.8$ [Fig. 4(b)], a result that differs significantly from the one found by us, due to the different boundary conditions adopted. However, it must be stressed here that both our results and those of Shadid and Goldstein have been obtained with poorly conducting boundaries, a configuration that differs significantly from the one where the boundaries conduct heat well, a condition that has been investigated extensively, both theoretically and experimentally for $\operatorname{Pr}=1.07$ [8,9,16,17]. Experiments and theoretical models performed with perfectly conducting 


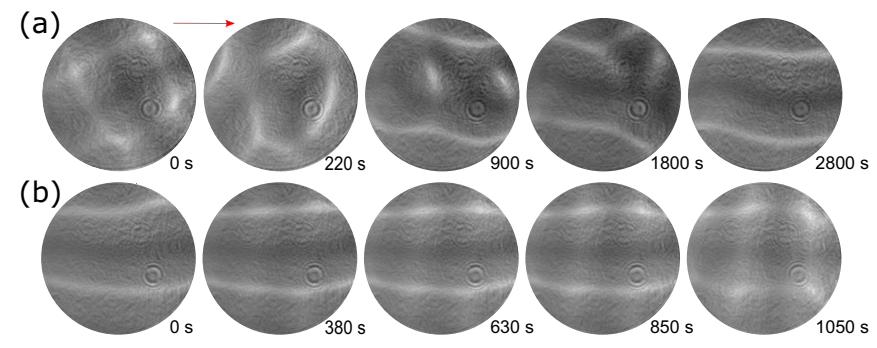

FIG. 5. Time evolution of the transition between the square and longitudinal roll patterns. (a) Transition from square to longitudinal roll pattern; (b) transition from longitudinal roll to square pattern. Time $t=0$ corresponds to the instant where an inclination of 27 mrad was either imposed or removed from the layer of liquid to trigger a shear stress. During the measurements the Rayleigh number was kept at a constant value $\mathrm{Ra}=1800$. Each frame has a diameter of $27 \mathrm{~mm}$. The arrow marks the projection of the direction of steepest ascent of the boundaries.

boundaries and in the absence of inclination show that the convective planform is made by parallel rolls with wave number $k=3.117$. By tilting the liquid layer the rolls align parallel to the direction of inclination. As it will be discussed in more detail below, in contrast with our results, this reorientations of the rolls occurs in the absence of a threshold inclination.

\section{Square-Longitudinal Roll Transition}

To investigate the mechanism of the transition between square and longitudinal roll patterns, we recorded sequences of shadowgraph images by keeping the sample at a constant Rayleigh number and changing its inclination rapidly, in a time of the order of a few seconds. The sample was initially kept horizontal at $\mathrm{Ra}=1800$ [Fig. 5(a), 0 s] until a stable square pattern was obtained. The inclination was then suddenly changed from 0 to $27 \mathrm{mrad}$, and a first sequence of images was acquired until a stable roll pattern was obtained [Fig. 5(a), 220-2800 s). The inclination was then removed and a second sequence of images was acquired [Fig. 5(b)]. From the two image sequences it is apparent that the mechanism of the square-to-roll transition is different from the one of the roll-to-square transition. In the first case, a first phase of the transition involves the complete demolition of the square pattern, which subsequently reorganizes to form the roll one. In the second case, a transverse roll structure grows perpendicularly to the longitudinal one, leading to the formation of a square pattern aligned to the roll one. The asymmetry between the two transitions is also reflected by their dynamics. In fact, the transition from the square pattern to longitudinal rolls is about three times faster than the one from longitudinal rolls to the square pattern. In the range of Rayleigh numbers $1500 \leqslant \mathrm{Ra} \leqslant 2800$ that characterizes the transition we do not observe a significant variation of the timescales of the transition in the two directions. Conversely, when the inclination is applied the transition time appears to be affected by the depth of penetration into the longitudinal rolls region. More in detail, the sudden imposition or removal of a tilt angle of 56 mrad gives rise to transitions between squares and longitudinal rolls a factor 4.7 faster than the ones reported at an angle of $27 \mathrm{mrad}$. We point out that the timescales involved in these transitions are strongly affected by the path in the phase space followed to reach the initial and final state. This prevents a fully quantitative investigation of the kinetics of the transition, because it would be in principle affected by a huge number of parameters.

\section{DISCUSSION AND CONCLUSIONS}

The investigation of the effect of inclination on convection in single component fluids with $\operatorname{Pr} \approx 1$ in the presence of conducting boundaries has shown that this system exhibits a rich phase space, which can be roughly divided into two main regions close to the threshold for Rayleigh-Bénard convection [8]: (i) for inclinations smaller than $77.5^{\circ}$ the convective planform is made of longitudinal rolls. Longitudinal rolls are formed through the alignment of the roll pattern observed close to threshold in the absence of inclination, due to the symmetry breaking determined by the large scale flow induced by the inclination; (ii) drifting transverse rolls develop at inclination angles larger than $77.5^{\circ}$. Interestingly, the transition to randomly aligned rolls in the absence of inclination to longitudinal rolls in the presence of inclination is continuous, and occurs in the absence of a threshold inclination angle [8]. Conversely, our results in the presence of poorly conductive boundaries provide clear evidence of the presence of a transition between square and roll patterns determined by the inclination at a threshold inclination $\delta \approx 14 \mathrm{mrad}$.

At such small inclination angles the component of the acceleration driving the large scale flow is about two orders of magnitude smaller than the acceleration of gravity itself. The fact that such a small component of the acceleration of gravity can induce a transition between convective planforms is a unique feature of the presence of poorly conducting boundaries. In fact, poorly conducting boundaries are not able to dissipate effectively heat, and a small temperature perturbation inside the fluid can survive for a long time and drive the large scale flow effectively, even in the presence of a small inclination. More quantitatively, in the presence of highly conductive boundaries the conductive dissipation of temperature inhomogeneities with the wave number of the convective structures would occur almost instantaneously close to the boundaries, leading to isothermal boundary layers. Conversely, in the presence of poorly conducting boundaries the temperature distribution at their surfaces becomes highly inhomogeneous in the presence of convection. This effect has been demonstrated experimentally by Shadid and Goldstein by using a thermosensitive liquid crystal layer in contact with the sample [24]. Under these conditions, the conductive dissipation of the temperature inhomogeneities would require a conduction time of the order of $\tau_{c}=\left[\kappa_{w}(k h)^{2}\right]^{-1} \approx 53000 \mathrm{~s}$ $\approx 15 \mathrm{~h}$, and even a small inclination of the liquid layer can drive a LSF.

This condition is qualitatively similar to the one encountered in a binary liquid mixture with positive Soret coefficient heated from below [31-33]. In fact, in this system the boundaries are impermeable to the transport of mass, a condition that mirrors insulating boundaries in Rayleigh-Bénard convection. In the absence of inclination, square patterns can develop in a horizontal layer of a binary mixture [18,32]. When subjected to a small tilt of the order of a few milliradians a transition 
between the square pattern and a drifting centered-rectangular one occurs $[18,21]$. The drifting pattern is made by parallel rows of ascending and descending columns of fluid moving into opposite directions parallel to the inclination of the fluid layer, in a superhighway convection (SHC) configuration resembling alternate rows of traffic on a highway. The simulation of this kind of system by using Boussinesq Navier Stokes equations has shown that the large scale flow gives rise to the formation of a concentration gradient parallel to the direction of inclination, determined by the stratification of the concentration along the direction of inclination [20,34]. The periodic SHC solution found for a binary mixture is strictly related to the presence of this horizontal density gradient, which is not present in a single component fluid, and this explains why we do not observe these structures in a single component fluid.
A further understanding of pattern formation in inclined layers of fluid with poorly conducting boundaries would require the utilization of combinations of fluid samples and boundaries with a ratio $\zeta$ of thermal conductivities spanning several orders of magnitude. This kind of requirement is difficult to fulfill in real experiments, but could be implemented effectively by means of computer simulations.

\section{ACKNOWLEDGMENTS}

The authors acknowledge discussion with $F$. Scheffold. Work partially supported by the European Space Agency, CORA-MAP TechNES Contract No. 4000128933/19/NL/PG.
[1] M. Cross and P. Hohenberg, Rev. Mod. Phys. 65, 851 (1993).

[2] S. Chandrasekhar, Hydrodynamic and Hydromagnetic Stability (Oxford University Press, Oxford, UK, 1961).

[3] E. Bodenschatz, W. Pesch, and G. Ahlers, Annu. Rev. Fluid Mech. 32, 709 (2000).

[4] J. E. Hart, J. Fluid Mech. 47, 547 (1971).

[5] J. E. Hart, J. Fluid Mech. 48, 265 (1971).

[6] R. M. Clever and F. H. Busse, J. Fluid Mech. 81, 107 (1977).

[7] K. Fujimura and R. E. Kelly, J. Fluid Mech. 246, 545 (1993).

[8] K. E. Daniels, B. B. Plapp, and E. Bodenschatz, Phys. Rev. Lett. 84, 5320 (2000).

[9] P. Subramanian, O. Brausch, K. E. Daniels, E. Bodenschatz, T. M. Schneider, and W. Pesch, J. Fluid. Mech. 794, 719 (2016).

[10] F. H. Busse and R. M. Clever, Phys. Fluids 12, 2137 (2000).

[11] K. E. Daniels and E. Bodenschatz, Phys. Rev. Lett. 88, 034501 (2002).

[12] G. Seiden, S. Weiss, J. H. McCoy, W. Pesch, and E. Bodenschatz, Phys. Rev. Lett. 101, 214503 (2008).

[13] K. E. Daniels, O. Brausch, W. Pesch, and E. Bodenschatz, J. Fluid. Mech. 597, 261 (2008).

[14] A. Zebib and M. M. Bou-Ali, Phys. Rev. E 79, 056305 (2009).

[15] O. Zier, W. Zimmermann, and W. Pesch, J. Fluid. Mech. 874, 76 (2019).

[16] F. Reetz and T. Schneider, J. Fluid Mechanics 898, A22 (2020).

[17] F. Reetz, P. Subramanian, and T. Schneider, J. Fluid Mechanics 898, A23 (2020).
[18] F. Croccolo, F. Scheffold, and A. Vailati, Phys. Rev. Lett. 111, 014502 (2013).

[19] M. Italia, F. Croccolo, F. Scheffold, and A. Vailati, Eur. Phys. J. E 37, 101 (2014).

[20] A. Alonso, I. Mercader, and O. Batiste, Phys. Rev. E 97, 023108 (2018).

[21] F. Croccolo, S. Castellini, F. Scheffold, and A. Vailati, Phys. Rev. E 98, 063104 (2018).

[22] I. Mercader, O. Batiste, A. Alonso, and E. Knobloch, Phys. Rev. E 99, 023113 (2019).

[23] P. Le Gal and V. Croquette, Phys. Fluids 31, 3440 (1988).

[24] J. Shadid and R. Goldstein, J. Fluid Mech. 215, 61 (1990).

[25] F. F. Busse and N. Riahi, J. Fluid Mech. 96, 243 (1980).

[26] M. R. E. Proctor, J. Fluid Mech. 113, 469 (1981).

[27] D. R. Jenkins and M. R. E. Proctor, J. Fluid. Mech. 139, 461 (1984).

[28] N. Riahi, J. Fluid. Mech. 152, 113 (1985).

[29] G. S. Settles, Schlieren and Shadowgraph Techniques: Visualizing Phenomena in Transparent Media, 1st ed., Experimental Fluid Mechanics (Springer-Verlag, Berlin/Heidelberg, 2001).

[30] F. Croccolo and D. Brogioli, Appl. Optics 50, 3419 (2011).

[31] C. Jung, B. Huke, and M. Lücke, Phys. Rev. Lett. 81, 3651 (1998).

[32] E. Moses and V. Steinberg, Phys. Rev. Lett. 57, 2018 (1986).

[33] P. Le Gal, A. Pocheau, and V. Croquette, Phys. Rev. Lett. 54, 2501 (1985).

[34] I. Mercader, O. Batiste, and A. Alonso, Comput. Fluids 39, 215 (2010). 